\title{
Local refinement based on the 7-triangle longest-edge partition
}

\author{
Ángel Plaza ${ }^{\mathrm{a}, *}$, Alberto Márquez ${ }^{\mathrm{b}}$, Auxiliadora Moreno-González ${ }^{\mathrm{b}}$, José P. Suárez $^{\mathrm{c}}$ \\ a Department of Mathematics, ULPGC, 35017-Las Palmas de Gran Canaria, Spain \\ ${ }^{\mathrm{b}}$ Department of Mathematics, University of Seville, Spain \\ ${ }^{\mathrm{c}}$ Department of Cartography and Graphic Engineering, ULPGC, Spain
}

Available online 31 January 2009

\begin{abstract}
The triangle longest-edge bisection constitutes an efficient scheme for refining a mesh by reducing the obtuse triangles, since the largest interior angles are subdivided. In this paper we specifically introduce a new local refinement for triangulations based on the longest-edge trisection, the 7-triangle longest-edge (7T-LE) local refinement algorithm. Each triangle to be refined is subdivided in seven sub-triangles by determining its longest edge. The conformity of the new mesh is assured by an automatic point insertion criterion using the oriented 1-skeleton graph of the triangulation and three partial division patterns.
\end{abstract}

(C) 2009 IMACS. Published by Elsevier B.V. All rights reserved.

Keywords: Local refinement; Longest-edge based algorithms; Skeleton

\section{Introduction}

In the last years many different strategies have been developed to locally refine triangular and tetrahedral meshes. There are two main steps in local adaptive refinement: the refinement of a subset of elements based on local error indicators, and the consistent transition between refined and unrefined cells [1,3]. We refer to the latter as 'mesh conformity'. Several refinement and improvement techniques for two- and three-dimensional triangulations are available $[2,5,13,4,7]$. Some of them are based on edge bisection of the elements, triangles or tetrahedra.

In two dimensions, the 4-Triangle Longest-Edge (4T-LE) partition and the associated local refinement was introduced by Rivara in [13,16,14]. This partition was extended to tetrahedral meshes in [8], where the tetrahedral mesh is locally refined after the skeleton of the triangulation, that is, the set of triangular faces of the tetrahedra, has been divided by the 4T-LE partition. The 4T-LE partition of a given triangle $t$ never produces an angle smaller than half the minimum original angle [18], and besides, it shows a remarkable mesh quality improvement [16] between certain limits, as recently studied in [12]. In order to devise a new strategy to improve mesh quality through iterative refinement, in this paper we use a new triangle partition, the seven-triangle longest-edge (7T-LE) partition [6]. This partition, first, positions two equally spaced points per edge and, then, the interior of the triangle is divided into seven sub-triangles in a manner compatible with the subdivision of the edges. Three of the new sub-triangles are similar to the original, two are similar to the new triangle also generated by the 4T-LE, and the other two triangles are, in general, better shaped.

\footnotetext{
* Corresponding author. Tel.: +34928 458827; fax: +34 928458711.

E-mail address: aplaza@dmat.ulpgc.es (Á. Plaza).
} 


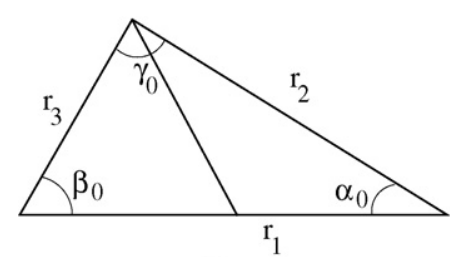

(a)

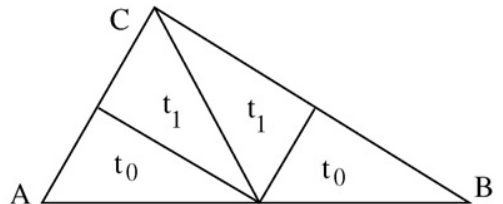

(b)

Fig. 1. (a) LE partition of triangle $t_{0}$ and (b) 4T-LE partition of triangle $t_{0}$.

The outline of this paper is as follows: Section 2 summarizes the 4T-LE partition and its improvement property, showing the limitations of this improvement. Section 3 presents the new 7T-LE partition and shows in a geometric diagram that the new triangles generated by the 7T-LE partition are better shaped that those generated by the 4T-LE partition. Section 4 introduces the new local refinement algorithm based on the 7T-LE partition, by using the 1-skeleton oriented graph approach [20]. Also the inverse counterpart algorithm for derefining a sequence of triangulation is presented. The paper closes with some conclusions and ongoing research.

\section{The 4T-LE partition and its improvement property}

Here the 4-triangle longest-edge partition and its self-improvement is summarized. The section closes with an example illustrating the limits of the characteristic improvement.

In the following, for any triangle $t$, the edges and angles will be respectively denoted in decreasing order $r_{1} \geq r_{2} \geq r_{3}$, and $\gamma \geq \beta \geq \alpha$. Furthermore, $t(\alpha, \beta, \gamma)$ will be the class of similar triangles with angles $\gamma \geq \beta \geq \alpha$. Interchangeably, $t$ will represent an element of the class $t \in t(\alpha, \beta, \gamma)$ or the class itself.

Definition 1. (edge bisection and longest-edge bisection) The longest-edge (LE) partition of a triangle $t_{0}$ is obtained by joining the midpoint of the longest edge of $t_{0}$ with the opposite vertex (Fig. 1 (a)). The 4-triangle longest-edge (4T-LE) partition is obtained by joining the midpoint of the longest edge to the opposite vertex and to the midpoints of the two remaining edges (see Fig. 1(b)).

Note that the two subtriangles with edges coincident with the longest edge of the parent are similar to the parent. The remaining two subtriangles form a similar pair that, in general, are not similar to the parent triangle. We refer to such new triangle shapes as 'dissimilar' to those preceding.

Since the first 4T-LE partition of any triangle $t_{0}$ introduces two new edges parallel to the edges of $t_{0}$, the first 4T-LE partition of a single triangle $t_{0}$ produces two triangles similar to $t_{0}$ and two (potentially) new similar triangles $t_{1}$; and, consequently, the iterative 4T-LE partition of any triangle $t_{0}$ introduces (at most) one new dissimilar triangle per iteration [16].

The iterative 4-triangle longest-edge partition produces a finite sequence of 'better' triangles satisfying the properties illustrated in the following diagram until triangle $t_{N}$ becomes non-obtuse, where $\alpha_{I}$, and $\gamma_{I}$ are respectively the smallest and the largest angles of triangle $t_{i}$. The arrow emanating from triangle $t_{i}$ to triangle $t_{i+1}$ means that the (first) 4-triangle longest-edge partition of triangle $t_{i}$ produces the new dissimilar triangle $t_{i+1}$ :

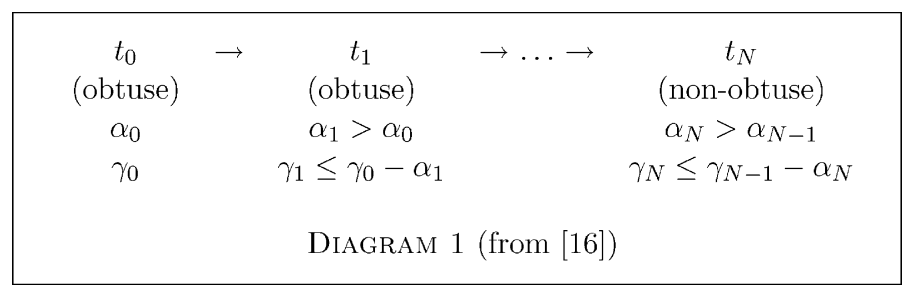

We refer the reader to [12] in which the self-improvement property of the 4T-LE partition has been studied in detail. By way of example, consider the initial triangle $t_{0}$ with angles $(\alpha, \beta, \gamma)=(1.95,32.595,145.455)$. The evolution of the smallest angle and largest angle for each of the new triangles generated by the 4T-LE partition is shown in Fig. 2. 


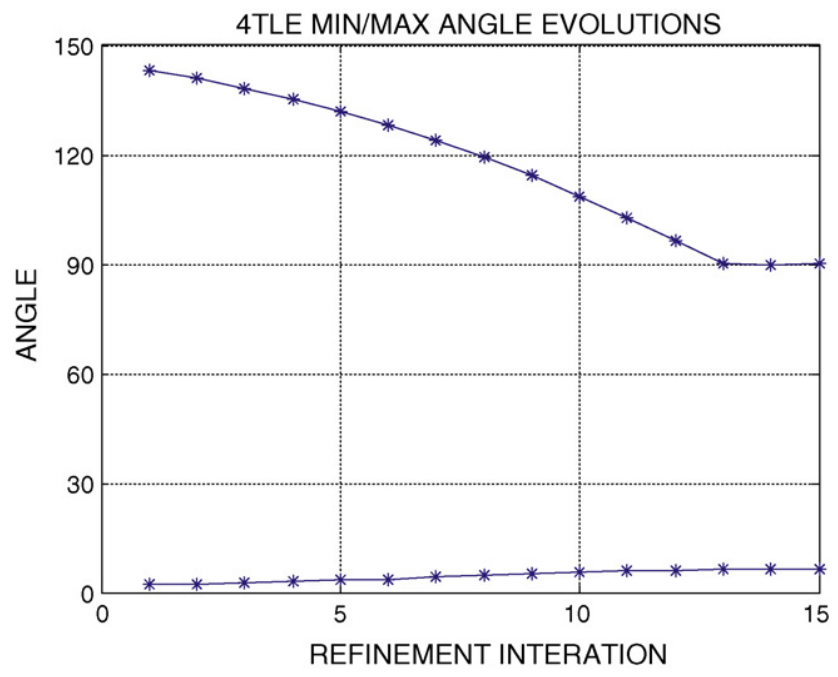

Fig. 2. Evolution of minimum and maximum angles by the 4T-LE partition of an initial triangle through iterative refinement.

Therefore, the self-improvement property of the 4T-LE partition, as proved both experimentally and mathematically in [12], needs to be handled with caution as this example clearly demonstrates.

Since the 4T-LE partition is based on the bisection of the edges, by locating midpoints on the edges of the triangle, we have introduced here a new triangular partition based on the trisection of the edges at equally spaced points. Our goal is to use this partition to introduce a new local refinement algorithm.

\section{The 7-triangle longest-edge partition}

In this section, a new longest-edge partition is presented. This partition is based on the trisection of the edges of the triangle, instead of the bisection of the edges as the 4T-LE.

Definition 2. (7-triangle longest-edge partition) The 7-triangle longest-edge (7T-LE) partition of a triangle $t_{0}$ is obtained as follows:

1. Position two equally spaced points per edge and join them, using parallel segments, to the edges, at the points closest to each vertex (see Fig. 3 (a)).

2. Join the two interior points of the longest edge of the initial triangle to the base points of the opposite sub-triangle in such a way that they do not intersect (see Fig. 3(b)).

3. Triangulate the interior quadrangle by the shortest diagonal (see Fig. 3(c)).
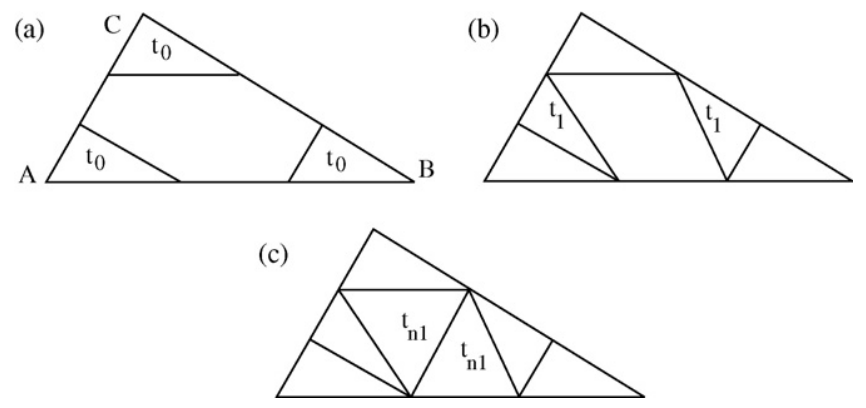

Fig. 3. 7T-LE partition of triangle $t_{0}$ : (a) edge trisection and cut off the corner triangles by parallel segments to the edges, (b) interior points of the $\mathrm{LE}$ are connected to the base points of opposite triangle and (c) triangulation of the interior quadrangle by the shortest diagonal. 


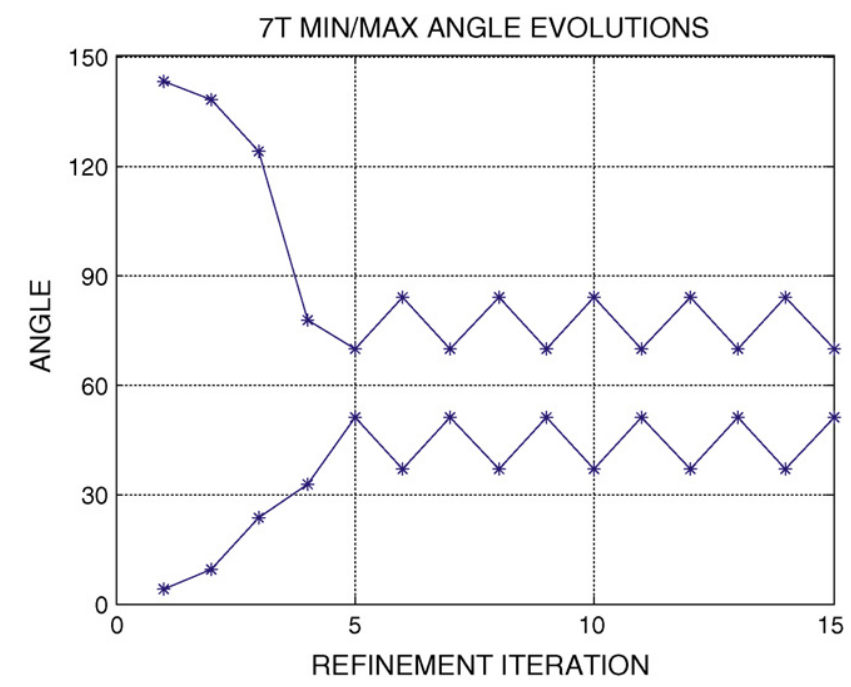

Fig. 4. Evolution of minimum and maximum angles by the 7T-LE partition.

It should be noted that, due to parallelism, the first three sub-triangles obtained are similar to the initial one $\left(t_{0}\right)$, whereas the second two sub-triangles are similar to the first-class Rivara triangle $\left(t_{1}\right)$. Finally, the last two triangles are not given with the 4T-LE Rivara partition and, consequently, will be called here, $t_{n 1}$. Note also that the area of sub-triangles $t_{0}$ and $t_{1}$ is $1 / 9$ of the area of the initial triangle, whereas the area of each sub-triangle $t_{n 1}$ is $2 / 9$ of the area of the initial triangle.

In order to compare the evolution of new triangles for the 7T-LE partition we consider the same initial triangle as before, that is $t_{0}$ with angles $(\alpha, \beta, \gamma)=(1.95,32.595,145.455)$. The evolution of the smallest angle and largest angle for each of the new triangles generated by the 7T-LE partition is shown in Fig. 4. It should be noted that the new triangles are better shaped that those produced by the 4T-LE partition.

Basing our hypotheses on the trisection of the edges, some other partitions may be considered. We present here one of these possibilities, also based on a local Delaunay triangulation:

Definition 3. (7-triangle Delaunay partition) The 7-triangle Delaunay (7T-D) partition of a triangle $t_{0}$ is obtained as follows:

1. Position two equally spaced points per edge.

2. Triangulate the cloud of points using the Delaunay triangulation (see Fig. 5(b)).

It should be noted that the 7T-D partition is not equivalent to the 7T-LE partition since the division of the interior hexagon depends upon the specific distribution of the distances between the vertices (see Fig. 5).

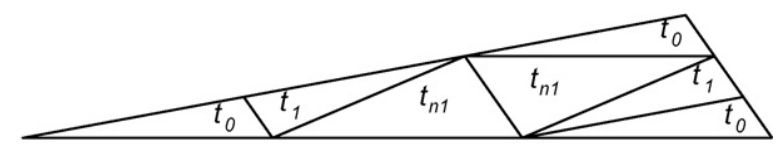

(a) 7T-LE partition of $t_{0}$

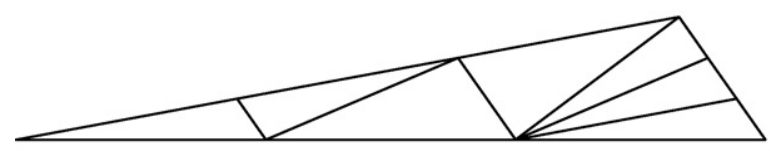

(b) 7T-D partition of $t_{0}$

Fig. 5. Two different 7T partitions of an obtuse triangle $t_{0}$. 


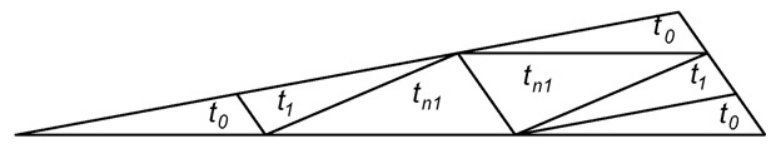

(a) 7T-LE partition of $t_{0}$

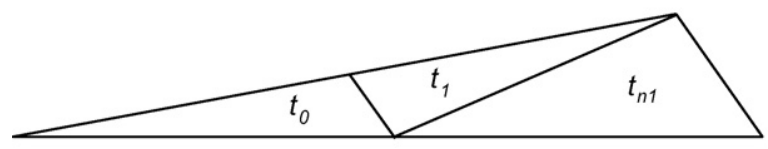

(b) 3T-LE partition of $t_{0}$

Fig. 6. 7T-LE partition and 3T-LE partition of an obtuse triangle $t_{0}$.

\subsection{Mesh quality improvement for the 7T-LE partition}

Note that the new triangle $t_{n 1}$ generated by the 7T-LE partition may also be seen as one of the triangles generated by the 3T-LE partition division, used in the local refinement associated with the 4T-LE partition (see Fig. 6).

Lemma 4. The number of dissimilar triangles arising in the iterative application of the 7T-LE partition to any initial triangle $t_{0} \equiv \triangle A B C$ is bounded.

See reference [6] for a proof of this result, and for a discussion and comparison between partition 4T-LE and 7T-LE depending on the shape of the initial triangle.

In order to experimentally show the result of previous Lemma, we use here a geometrical diagram introduced in [10]. For a given triangle the longest edge is scaled to have unit length. It follows that the set of all triangles is bounded by this horizontal segment (longest edge) and by two bounding exterior circular arcs of unit radius. This mapping domain comprised by the horizontal segment of extreme points $(0,0)$ and $(1,0)$, the unit circular arcs which center at these extreme points, allows us to represent the shape evolution for the new triangles generated by iterative application of any triangle partition.

Here we carried out the following computational experiment. Select a point $(x, y)$ within the mapping domain. Point $(x, y)$ defines the apex of a target triangle with additional vertices at $(0,0)$ and $(1,0)$. For this triangle, 7T-LE division is successively applied as long as a new dissimilar $t_{n 1}$ triangle appears. This means that we recursively apply 7 T-LE and stop when the shapes of new generated triangles are the same as those already generated in previous refinement steps. The number of such refinements to reach termination defines the number of dissimilar triangles associated with the initial triangles associated with the initial triangle and this numerical value is assigned to the initial point $(x, y)$ chosen. This process is progressively applied to a large sample of triangles (points) uniformly distributed over the domain. Finally, we graph the respective values of dissimilar triangles in a corresponding color map to obtain the result in Fig. 7. The same experiment for the 4T-LE partition is shown in Fig. 8.

It should be noted that the dark blue region in the previous figure corresponds to the region in which all the trajectories end, with total independence of the initial position. Therefore, for the 7T-LE partition, a lower bound for the maximum of the smallest angles for triangles $t_{n 1}$ is $\alpha=30^{\circ}$ corresponding to the apex with $x=1 / 4$, or the apex with $x=3 / 4$. Note also that the smallest angle in each of the regions generated in this way, is bounded from below with total independence of the initial point of the respective trajectories. This is a remarkable feature in comparison with the evolution of the angles for the 4T-LE partition. In the 4T-LE partition these lower bounds depend on the geometry of the initial triangle. See [12] for details, and observe, for instance, Fig. 9 left.

For a better understanding of the self-improvement property of the 4T-LE partition and the limits of this property as given in this paper, the successive triangles obtained have been normalized to share the longest edge. The various triangles obtained in the first test problem are depicted in Fig. 9.

The evolution of the new triangles generated by the 7T-LE partition vs. the triangles generated by the 4T-LE partition can be observed, at a first glance, from the diagrams in Fig. 10. There, the apexes of the triangles have been numbered in the order in which they have been generated. Apexes of triangles generated by the 4T-LE partition are joined by a 


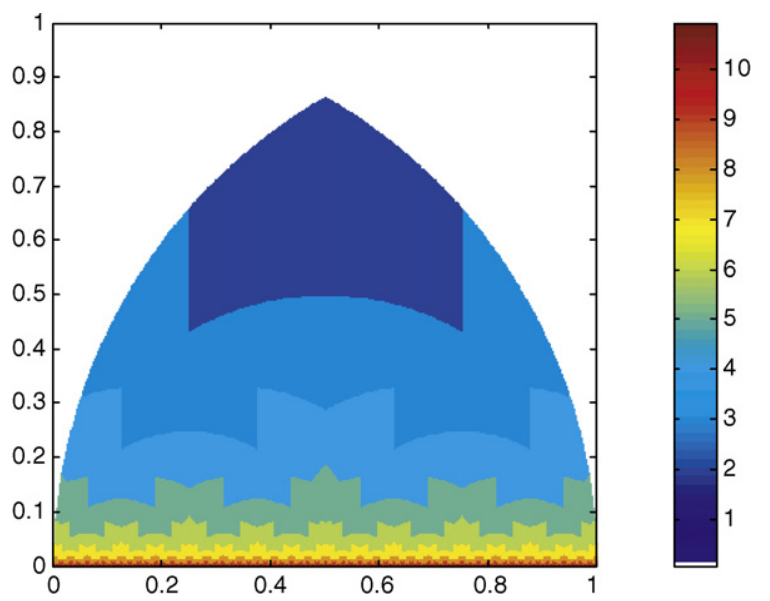

Fig. 7. Color diagram representing triangle classes generated by a Montecarlo experiment for the 7T-LE partition.

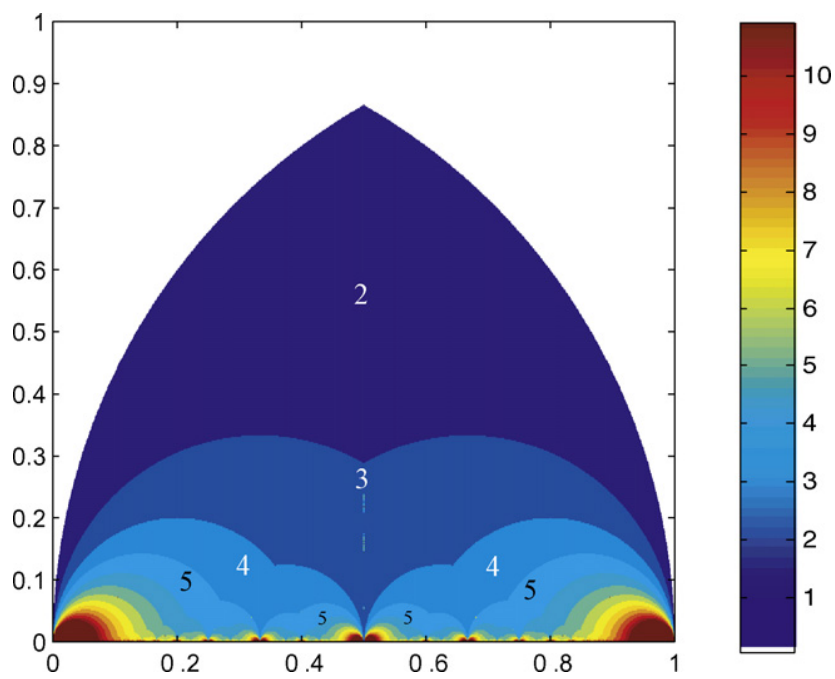

Fig. 8. Dissimilar triangle classes generated by computer experiment for the 4T-LE partition.
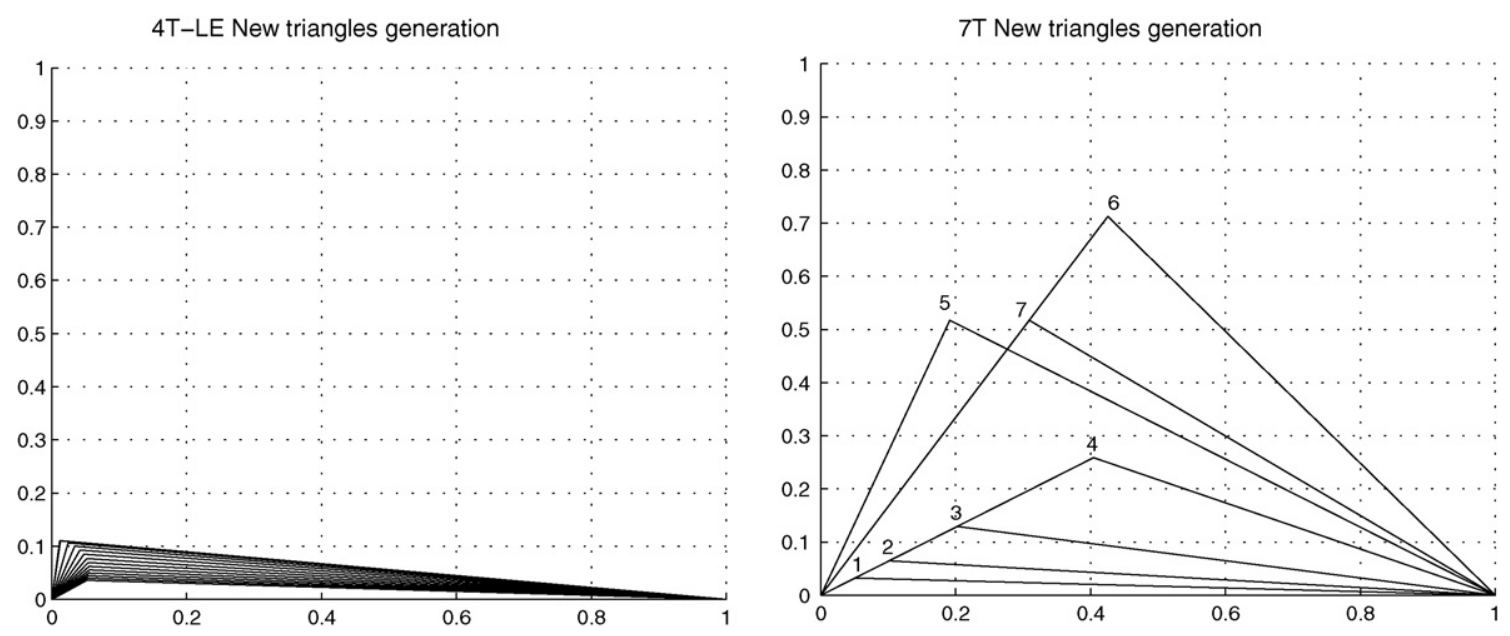

Fig. 9. Evolution of dissimilar triangles of example test 1. 

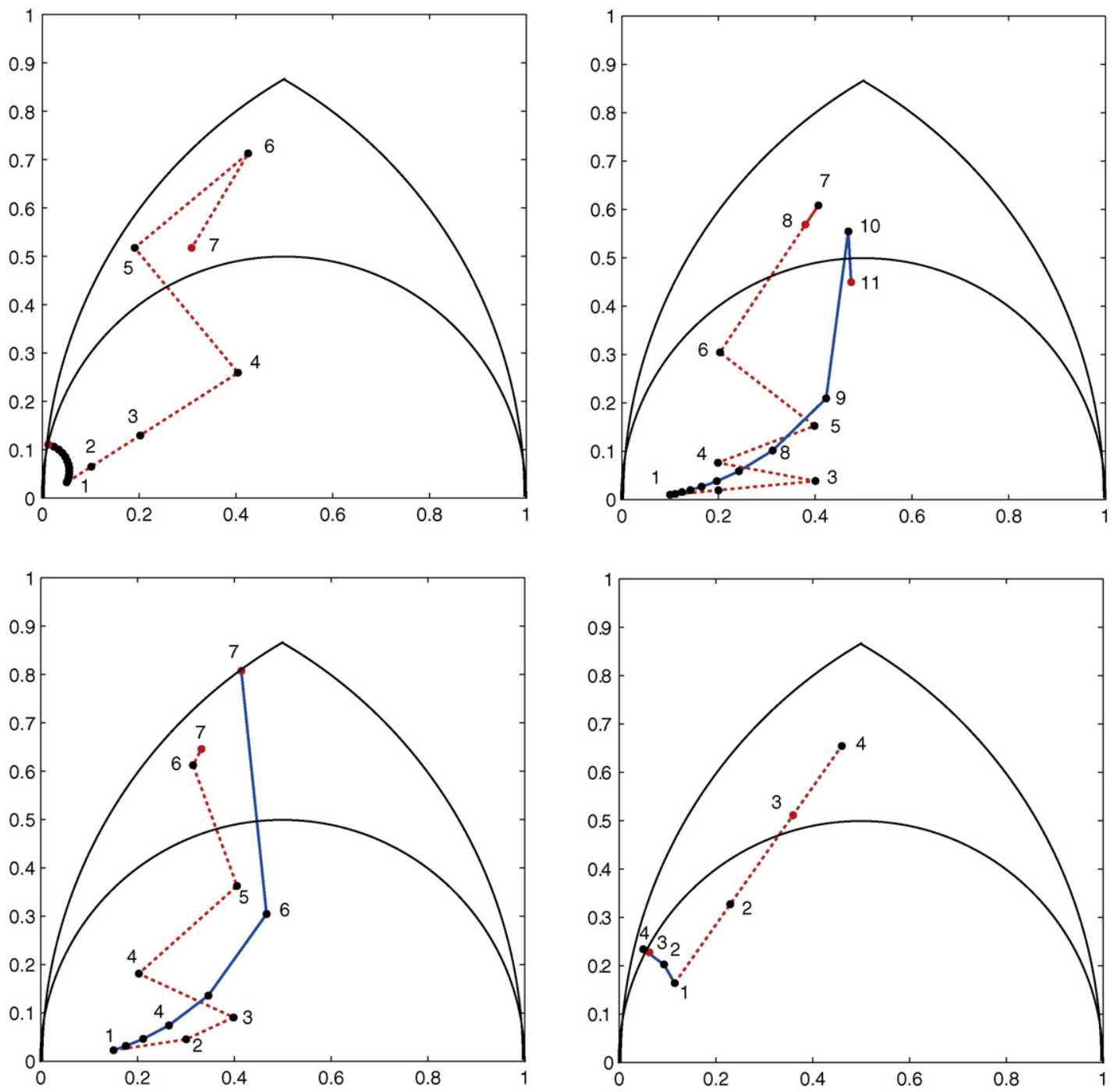

Fig. 10. Evolution of the triangles given by the 4T-LE partition (bold line) vs. 7T-LE partition (dashed line) for different initial triangles.

bold line, while the apexes of the new triangles coming from the 7T-LE partition are jointed by a dashed line. Note, that in general, triangles on the dashed line are better shaped that the corresponding triangles on the bold line.

\section{Local refinement algorithm based on the 7T-LE partition}

Similarly to the local refinement based on the 4T-LE partition, we present here a local refinement based now on the 7T-LE partition. That is, the particular triangles to be refined the 7T-LE partition is chosen for dividing. Then, in order to assure the conformity of the arising new mesh we use one of the partial partitions given in Fig. 11, where for comparison the partial partitions used by the local refinement based on the 4T-LE partition are also shown. Observe that 4T-LE partitions used 1 midpoint per edge subdivided, while 7T-LE partition used two interior, equally spaced, points per edge subdivided.

In the local refinement algorithm presented here we use the concept of 1-skeleton, see reference [20] for details.

Here we use the concepts of longest-edge propagation path (LEPP) and 1-skeleton of a two-dimensional triangulation. The (LEPP) of a triangle $t$ into a given triangulation as defined by Rivara [14] is the ordered list of triangles $\left\{t=t_{0}, t_{1}, \ldots, t_{n}\right\}$ such that triangle $t_{i}$ is the neighbor triangle of $t_{i-1}$ adjacent to the longest-edge of $t_{i-1}$. 


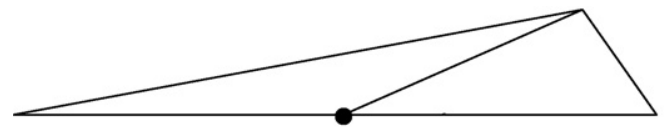

(a) $1 \mathrm{P} \times \mathrm{LE}$

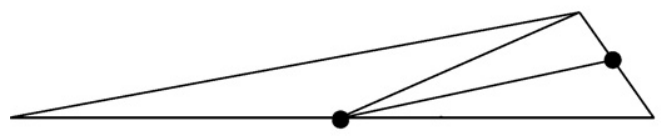

(b) $1 \mathrm{P} \times L E+1 \mathrm{P} \times \mathrm{SE}$

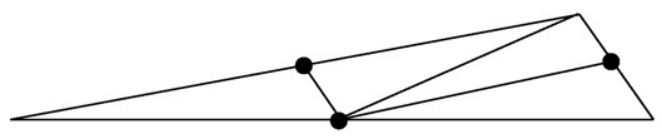

(c) $1 \mathrm{P} \times L E+1 \mathrm{P} \times M E$

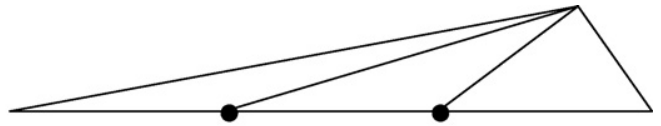

(a) $2 \mathrm{P} \times \mathrm{LE}$

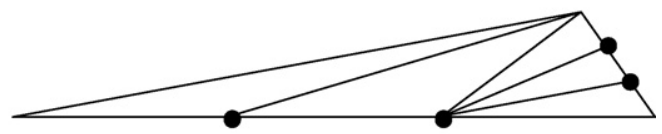

(b) $2 \mathrm{P} \times \mathrm{LE}+2 \mathrm{P} \times \mathrm{SE}$

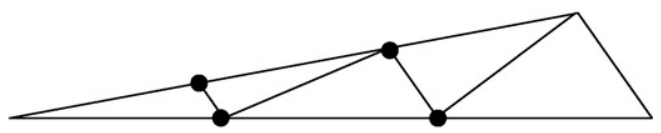

(c) $2 \mathrm{P} \times \mathrm{LE}+2 \mathrm{P} \times \mathrm{ME}$

Fig. 11. Partial partitions of a triangle depending on the number of edges subdivided for the 4T-LE local refinement (left) and for the 7T-LE local refinement (right).

On the other hand for a triangulation $\tau$ its 1-skeleton is the undirected graph $G^{1}(\tau)=G^{1}(P, L)$, where $P$ is the set of edges of $\tau$ and $L$ represents the topological adjacency relationship between two different nodes (edges) of $P$. So two edges $A$, and $B$ are connected in $G^{1}(P, L)$, that is $\exists l \in L$ such that $l \approx(A, B)$ if they share a extreme node in $\tau$, that is, if $A \cap B \neq \emptyset$. The edges of any triangle may be classified into two types. The longest-edge will be edge type 1 , and the other two edges will be type 2 . This classification induces an order into the 1-skeleton graph by considering the relation $\mathbf{R}=$ 'length of edge $x$ in the triangle is less than length of edge $y^{\prime}$. This order relation induces an orientation into the graph representing such order. See Fig. 12 for the 1-skeleton (oriented) graph associated to a single triangle (a) and to a whole triangulation (b). Let $t_{0}$ a triangle in a given triangulation $\tau$, and $e_{i}$ an edge of $t_{0}$. We will denote by LEPP $\left(e_{i}, G^{1}(\tau)\right)$ the LEPP of the neighbor triangle to $t_{0}$ sharing with $t_{0}$ edge $e_{i}$.

The refinement of triangular meshes involves two main tasks. The first is the subdivision of the target triangles and the second is the propagation to successive neighbor triangles to preserve conformity. If the refinement is uniform then all triangles in the mesh are refined and no propagation is induced on the mesh. Refinement can also be used locally for a small group of triangles in a mesh and in this case a neighbor triangle refinement (propagation) is
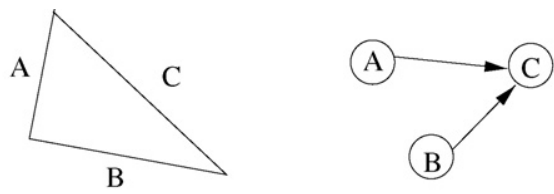

(a)
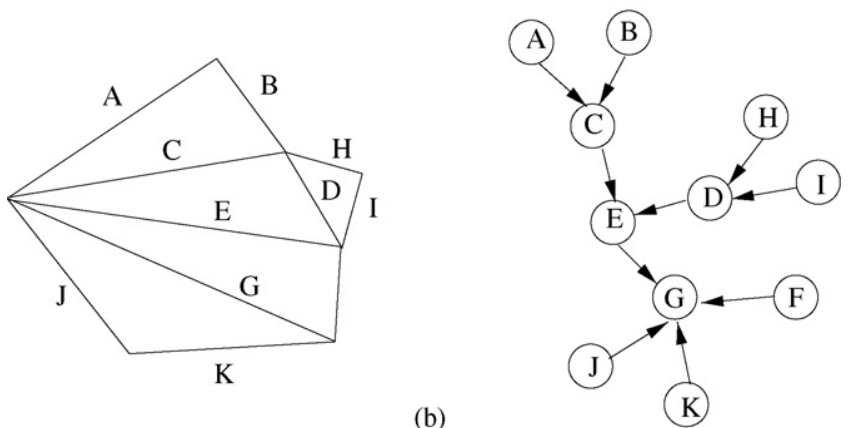

(b)

Fig. 12. 1-skeleton oriented graph for a single triangle (a) and for a whole triangulation (b). 
induced in the mesh to preserve conformity. In the LE schemes the longest edge of triangle $t$ is first bisected (or trisected) to form two (or three) subtriangles. Then, to preserve mesh conformity, the refinement is continued to the associated edge neighbor using a longest-edge strategy until the propagating path terminates. In the 4T-LE and also for the 7T-LE scheme, LE refinement propagates through all three neighbors triangles of $t$ as in the basic LE scheme preceding. The refinement may also be viewed as follows: (1) identify and divide by trisection the edges specified by the overall refinement indicators (steps 1 and 2 in the scheme of the algorithm bellow) and (2) subdivide the individual triangles to define the new triangulation (step 3). Concerning computational efficiency, it should be noted that the 7T-LE algorithm is of linear complexity with respect to the number of nodes. In this sense, from the computational efficiency point of view, the 4T-LE and the 7T-LE algorithms are equivalent. However, as can be observed in the example bellow, the 7T-LE algorithm produces denser meshes for the same refinement steps.

It is interesting to note that if $G^{1}$ is the 1-skeleton directed graph of a 2D conforming triangulation $\tau$, then $G^{1}$ does not contain cycles [20]. In addition, updating the 1-skeleton directed graph data structure by applying 4T-LE (and also the 7T-LE) partition affects only up to three graph nodes in each refinement step. Note that from a computational point of view, this fact ensures the locality of the 1-skeleton directed graph data structure.

$$
\begin{aligned}
& \text { Algorithm Local Refinement 7T-LE }\left(\tau, t_{0}\right) \\
& /^{*} \text { Input: } \tau \text { mesh, } t_{0} \text { triangle to be divided } \\
& /^{*} \text { Output: } \tau \text { mesh } \\
& \text { 1. } L=1-\operatorname{skeleton}\left(t_{0}\right)
\end{aligned}
$$

For each edge $e_{i} \in L$ do

$$
\operatorname{Trisection}\left(e_{i}\right)
$$

End

2. For each edge $e_{i} \in L$ do

$$
\begin{aligned}
& S_{i}=L E P P\left(e_{i}, G^{1}(\tau)\right) \text { do } \\
& \text { For each triangle } t_{i} \in S_{i} \text { do } \\
& \qquad \text { Let } e_{i} \text { be the longest edge of } t_{i} \\
& \quad \text { Trisection }\left(e_{i}\right) \\
& \text { End }
\end{aligned}
$$

End

3. For each triangle $t_{j} \in \tau$ to be subdivided do

$\operatorname{Subdivision}\left(t_{j}\right)$

End

An example of local refinement based on the 7T-LE partition is presented in Fig. 13. In both cases only a small region is chosen to be subdivided by the 4T-LE partition, and by the 7T-LE partition. Notice that by the 7T-LE procedure a more localized refinement is achieved in fewer refinement steps. For a comparison of the quality measurement evolution when each partition is locally applied, the mean value of the quality measurement $\eta$ has been used in this example. Bearing in mind that for each triangle $t$, the quality measurement $\eta$ is defined by

$$
\eta(t)=\frac{4 A \sqrt{3}}{l_{1}^{2}+l_{2}^{2}+l_{3}^{2}}
$$




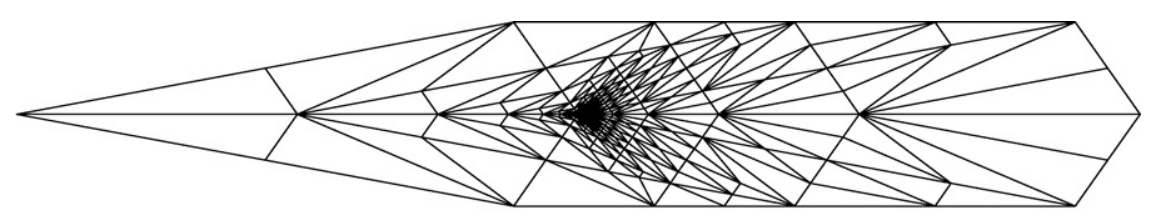

(a) 4T-LE partition. 1, 826 triangles after 33 refinement steps

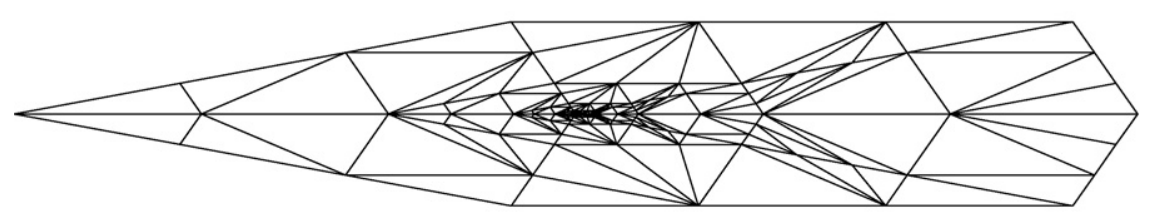

(b) 7T-LE partition. 1, 838 triangles after 28 refinement steps

Fig. 13. Example of local refinement. Based on the 4T-LE partition and based on the 7T-LE partition (a) 4T-LE partition. 1826 triangles after 33 refinement steps. (b) 7T-LE partition. 1838 triangles after 28 refinement steps.

where $A$ is the area of $t$, and $l_{1}, l_{2}$, and $l_{3}$ the lengths of the sides of the triangle. For the equilateral triangle $\eta=1$ where $l_{1}=l_{2}=l_{3}=\sqrt{(4 A \sqrt{3} / 3)}$. It should also be noted that other quality measurements may be used for this purpose, but they give similar patterns [6].

Fig. 14 shows the evolution of the average of $\eta(t)$ for the triangles $t$, at each mesh level, when the two reference partitions, 4T-LE, and 7T-LE are repeatedly applied locally. The quality evolution showed by the 7T-LE partition in this example is slightly better than that given with the 4T-LE partition. However, further research on the quality of the meshes obtained by local refinement is needed.

The fractal appearance of the adapted meshes in the neighborhood of the critical point is clear both for the 4T-LE local refinement and for the 7T-LE approach. In this sense is useful to use the concept of stable molecule associated to a node $P$ as follows [15,11].

Theorem 5 (Given any conforming triangulation). $\tau$, for any vertex $P$ of $\tau$, the arbitrary iterative use of the 7T-LE refinement algorithm to refine the triangulation around the vertex $P$, divides the angles converging in $P$ in a finite number of $k$ parts.

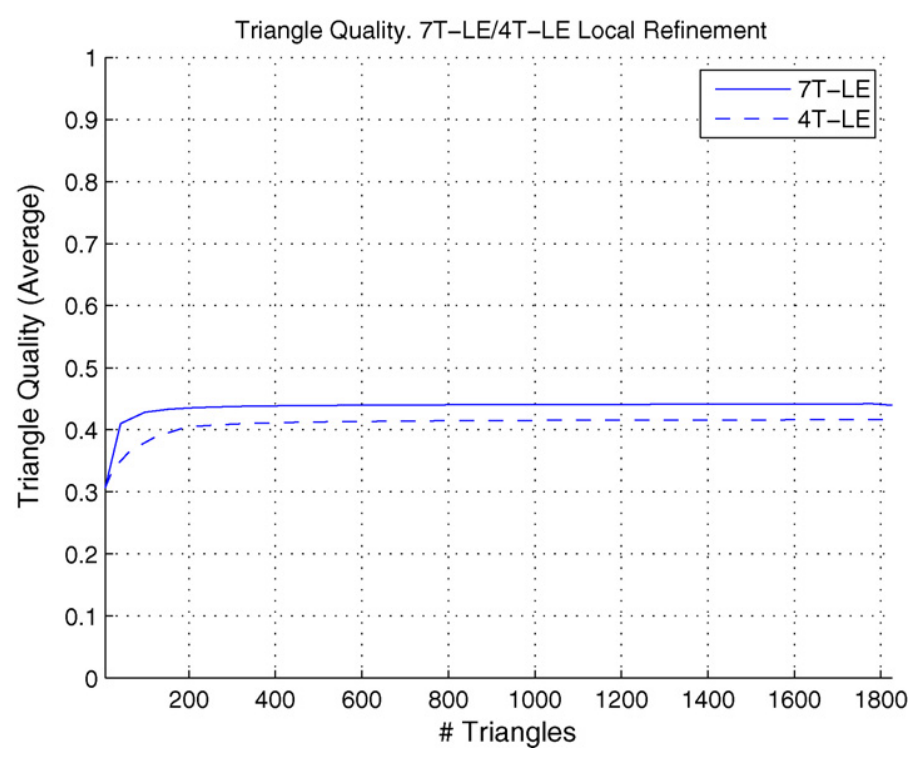

Fig. 14. Evolution of mesh quality (mean value of the quality measure for each triangle per refinement step). 


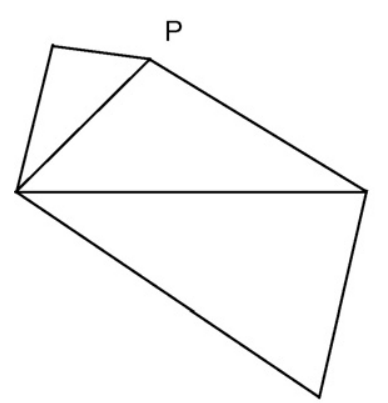

(a) Initial mesh

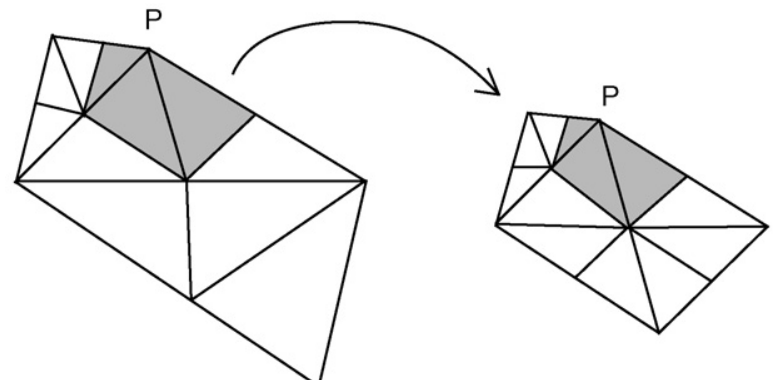

(b) 4T-LE refinement

(c) Further refinement

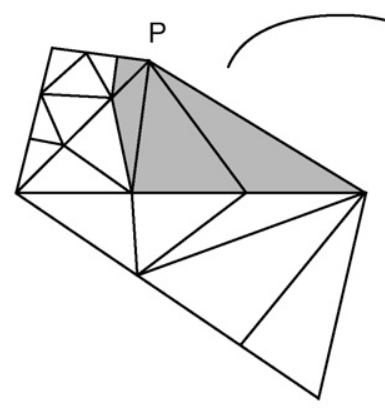

(b') 7T-LE refinement

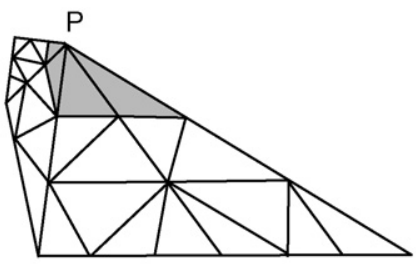

(c') Further refinement

Fig. 15. Stable molecule at point $P$ by the 4T-LE local refinement (b and c), and by the 7T-LE local refinement partition (b' and c').

Definition 6. (stable molecule) For any conforming triangulation $\tau$ and any vertex $P$ of $\tau$, the stable molecule associated with vertex $P$ is the partition of the plane around vertex $P$, induced by the use of some triangle refinement scheme of each triangle around the vertex $P$, such that further refinements of the triangles around $P$ do not change the number of triangles sharing $P$.

The following result holds:

Theorem 7. Let $\tau$ be any conforming triangulation and consider any vertex $P$ of $\tau$. The use of the 7T-LE refinement algorithm to refine the triangulation around the vertex $P$ produces triangulations that have the following characteristics: (a)After a finite number of iterations, the algorithm produces a triangulation $\tau^{*}$ such that the stable molecule associated with vertex $P$ is obtained. (b) The next iterations of the algorithm do not partition the angles of the stable molecule, but only introduce a set of new vertices distributed in geometric progression along the sides of the stable molecule of $P$.

An example of the stable molecule with vertex $P$ is shown in Fig. 15. Note that by using longest-edge based algorithms for refining, the stable molecule with vertex $P$ is achieved once the opposite edge to vertex $P$ is not longer the longest-edge of all the triangles sharing $P$. This explains previous Theorem.

\subsection{T-LE derefinement algorithm}

In should be noted here that the inverse counterpart algorithm for derefining a triangulation can be developed, in the same way that it has been introduced for the 4T-LE scheme [11]. To this end, the edges of a triangulation will be marked or unmarked respectively if their interior nodes remain or can be eliminated according to some derefinement criterion. In addition, if an edge must remain into the triangulation, this fact induces that some other edges have also to remain for conforming considerations. An outline of the 7T-LE derefinement algorithm is given below: 
7T-LE Derefinement Algorithm $(T, \epsilon)$

${ }^{*}$ Input: $T=\left\{\tau_{1}, \tau_{2}, \ldots, \tau_{k}\right\}, \epsilon=$ tolerance

/* Output: $T^{\prime}=\left\{\tau_{1}^{\prime}, \tau_{2}^{\prime}, \ldots, \tau_{k}^{\prime}\right\}$

For $i=k$ to 2 do

For each subdivided edge $e_{j} \in \tau_{i}$ do

If the interior nodes of $e_{j}$ must remain, then

$\operatorname{mark}\left(e_{j}\right)$

Assure conformity $\left(e_{j}\right)$

End

End

For $i=2$ to $k$ do

Re-triangulate mesh level $\tau_{i}$

End

Assure conformity $\left(e_{j}\right)$

$S_{j}=\operatorname{LEPP}\left(e_{j}, G^{1}(\tau)\right)$

For each triangle $t_{i} \in S_{j}$ do

Let $e_{i}$ be the longest edge of $t_{i}$

If the interior nodes of $e_{i}$ are unmarked, then

$\operatorname{mark}\left(e_{i}\right)$

Assure conformity $\left(e_{i}\right)$

End

End

It is worth noting that the derefinement condition is checked in a minimum number of nodes: only the interior nodes of eligible edges in each mesh-level, and out of these, only those suitable to be cancelled are taken for evaluation. If one node cannot be eliminated no neighboring nodes can be cancelled either.

Basically the derefinement algorithm proposed here is based into three main steps:

- Derefinement condition

- Assuring conformity

- Definition new connections

Conformity of the arising new mesh is assured in the same way that for the refinement procedure, that is, by using locally, for each remaining edge, the 1-skeleton oriented graph associated to the triangulation. Once all the edges have been evaluated every triangle of the preceding mesh level is taken to re-define the new connection of its descendants. This issue comprises two operations: clean up of the triangle and re-definition of the new connections. The second one is done by the same procedure used at refining. 
Finally, since the conformity is assured locally, and only the eligible edges at each level are chosen for evaluating the derefining condition, the derefining algorithm is linear in the number of edges.

\section{Conclusions and current research}

In this paper, the 7-triangle longest-edge (7T-LE) partition has been used to develop a new local refinement algorithm based on the trisection of the edges. The so-called self-improvement property of this partition has been shown numerically and compared with the same property of the 4-triangle longest-edge partition. The proposed 7T-LE refinement algorithm is an alternative algorithm for the local refinement problem. Finally the use of the LEPP concept along with the 1-skeleton of the triangulation allow us to implement a fully local 7T-LE algorithm, and also to implement its counterpart inverse derefinement algorithm.

Since the improvement of the triangulation is much better that for the 4T-LE algorithm in the case of obtuse triangles one could ask how to combine the proposed algorithm with other compatible partition for the case of acute triangles. Other related open problems, under further consideration are the following:

- Study of the propagation path due to the extension for conformity, in a similar way that it has been studied for the 4T-LE algorithm [21,22].

- Study of the number of dissimilar triangles generated by the iterative application of the 7T-LE partition [12].

- Study of a lower bound on the angles of triangles constructed by longest-edge trisection $[18,9]$.

- Study of a the convergence of the longest-edge trisection partition method for all the triangles [19].

- Use of longest-edge trisection techniques for the automatic refinement of Delaunay triangulations [15].

- Cost analysis of the conformity extension for the longest-edge trisection based refinement [17].

- Study of the mesh smoothness, that is if the transition between small and large elements is gradual depending on the particular triangular partition used. Smoothness is directly connected with the propagation problem in the local refinement of meshes.

\section{Acknowledgments}

This work has been partially supported by CICYT Project number MTM2005-08441-C02-02 and by CICYT Project number MTM2008-05866-C03-02/MTM, both from Ministerio de Ciencia e Innovación of Spain.

\section{References}

[1] I. Babuška, W. Rheinboldt, Error estimates for adaptive finite element computations, SIAM J. Numer. Anal. 15 (4) (1978) $736-754$.

[2] E. Bänsch, Local mesh refinement in 2 and 3 dimensions, Impact Comput. Sci. Eng. 3 (3) (1991) 181-191.

[3] G. Carey, Computational Grids: Generation, Adaptation and Solution Strategies, Taylor \& Francis, 1997.

[4] L. Freitag, Tetrahedral mesh improvement using swapping and smoothing, Int. J. Numer. Methods Eng. 40 (21) (1997) $3979-4002$.

[5] I. Kossaczký, A recursive approach to local mesh refinement in two and three dimensions, J. Comput. Appl. Math. 55 (3) (1994) $275-288$.

[6] A. Marquez, M. Moreno-González, A. Plaza, J. Suárez, The 7-triangle longest-side partition of triangles and mesh quality improvement, Finite Elem. Anal. Des. 44 (12-13) (2008) 748-758.

[7] M. Padrón, J. Suárez, A. Plaza, Refinement based on longest-edge and self-similar four-triangle partitions, Math. Comp. Simul. 75 (5-6) (2007) 251-262.

[8] A. Plaza, G. Carey, Local refinement of simplicial grids based on the skeleton, Appl. Numer. Math. 32 (2) (2000) $195-218$.

[9] A. Plaza, S. Falcón, J. Suárez, On the non-degeneracy property of the triangle longest-edge trisection, Appl. Math. Comp., in press.

[10] A. Plaza, J. Suárez, G. Carey, A geometric diagram and hybrid scheme for triangle subdivision, Comp. Aided Geom. Des. 24 (1) (2007) $19-27$.

[11] A. Plaza, J. Suárez, M. Padrón, Fractality of refined triangular grids and space-filling curves, Eng. Comp. 20 (4) (2005) $323-332$.

[12] A. Plaza, J. Suárez, M. Padrón, S. Falcón, D. Amieiro, Mesh quality improvement and other properties in the four-triangles longest-edge partition, Comput. Aided Geom. Des. 21 (4) (2004) 353-369.

[13] M.-C. Rivara, Algorithms for refining triangular grids suitable for adaptive and multigrid techniques, Int. J. Numer. Methods Eng. 20 (4) (1984) $745-756$.

[14] M.-C. Rivara, New longest-edge algorithms for the refinement and/or improvement of unstructured triangulations, Int. J. Numer. Methods Eng. 40 (18) (1997) 3313-3324.

[15] M.-C. Rivara, P. Inostroza, Using longest-side bisection techniques for the automatic refinement of delaunay triangulations, Int. J. Numer. Methods Eng. 40 (4) (1997) 581-597. 
[16] M.-C. Rivara, G. Iribarren, The 4-triangles longest-side partition of triangles and linear refinement algorithms, Math. omput. 65 (216) (1996) $1485-1502$.

[17] M.-C. Rivara, M. Vemere, Cost analysis of the longest-side (triangle bisection) refinement algorithm for triangulations, Int. J. Numer. Methods Eng. 12 (3-4) (1996) 224-234.

[18] I. Rosenberg, F. Stenger, A lower bound on the angles of triangles constructed by bisecting the longest side, Math. Comput. 29 (1975) $390-395$.

[19] M. Stynes, On faster convergence of the bisection method for all triangles, Math. Comput. 35 (152) (1980) 1195-1201.

[20] J. Suárez, G. Carey, A. Plaza, Graph-based data structures for skeleton-based refinement algorithms, Comm. Numer. Methods Eng. 17 (12) (2001) 903-910.

[21] J. Suárez, A. Plaza, G. Carey, The propagation problem in longest-edge refinement, Finite Elem. Anal. Des. 42 (2) (2005) $130-151$.

[22] J. Suárez, A. Plaza, G. Carey, Propagation of longest-edge mesh pattern in local adaptive refinement, Commun. Numer. Methods Eng. 24 (7) (2008) 543-553. 